\title{
L’esprit de compassion dans les soins infirmiers
}

\section{en oncologie}

Par Janice Post-White

\section{Abrégé}

Le cancer éveille en nous le besoin d'honorer notre être spirituel. $C$ 'est dans la résilience de l'esprit humain que les patients et les proches trouvent force et espoir et grâce à elle qu'ils poursuivent leur cheminement. Nous, les infirmières en oncologie, amenons les familles à prendre elles-mêmes le contrôle en cultivant notre essence intérieure et en la partageant avec eux, en honorant leur cheminement, en étant totalement présentes à leurs côtés et en guidant leurs pas à mesure qu'elles anticipent les défis à venir. L'esprit de compassion, ce n'est pas faire pour les autres, mais plutôt leur montrer que nous sommes à leurs côtés; c'est être le vent qui gonfle leurs voiles. Lorsque nous entrons en relation avec notre essence spirituelle, nous restons ouvertes au mystère de la vie. Invitez donc le mystère et la découverte dans votre vie afin de développer votre esprit de compassion et de ne pas oublier que cela vaut la peine de rechercher les arcs-en-ciel.

Remarque: Cet article est une version éditée de la conférence présentée dans le cadre des cérémonies d'ouverture de la conférence annuelle de l'ACIO 2002 à Winnipeg. Comme elle en a éliminé une grande partie des récits, l'auteure encourage les lecteurs et lectrices à puiser dans leur propre répertoire riche et varié de récits relatifs à la pratique clinique pour illustrer les concepts de spiritualité, de mystère, d'espoir et d'autonomisation.

Apprécier l'esprit de compassion dans les soins infirmiers en oncologie est un thème opportun pour moi alors que je passe six mois à Hawaii pour réfléchir à mes buts et à l'orientation de mes travaux de recherche et pour intégrer l'expérience du cancer qui frappe ma famille. En effet, cela fait deux ans que mon fils Brennan a fini son traitement pour sa leucémie aiguë lymphoblastique. La vie que nous menons sur une petite île isolée du Pacifique donne à chacun le temps et l'espace nécessaires à la réflexion, au ressourcement et à l'épanouissement spirituel.

Chaque jour, je m'émerveille devant le panorama immuablement beau. Sur l'île de Lanaï, je scrute patiemment les eaux de la baie Hulopoe dans l'espoir que les dauphins à long bec y feront une de leurs apparitions certes occasionnelles, mais si spectaculaires. Les visites des rorquals à bosse sont bien plus prévisibles puisqu'ils migrent chaque année vers les eaux chaudes des îles Hawaii pour la mise à bas. Et au moment où les feuilles commencent à tomber des arbres du Midwest américain et de la plupart des régions du Canada, les araignées des jardins commencent à tisser, comme toujours, leurs toiles en prévision d'un autre cycle des saisons. En Hawaii, les changements de saison sont moins spectaculaires. Ils sont si subtils qu'ils exigent toute notre attention.

Chaque nouvelle journée a son caractère prévisible. Tous les matins, le soleil se lève au-dessus d'îles volcaniques en sommeil, les petits oiseaux chantent entre eux lorsque la lumière du jour touche leur monde et les vagues viennent mourir sur le littoral volcanique. Les marées ne manquent jamais de monter et de descendre, deux fois par jour, tous les jours de l'année, et leur hauteur et celle des vagues est prévisible en fonction des phases de la Lune. La nuit, les étoiles affirment leur vue sur le monde, perchées si loin des terres où tout est assoupi. Cette prévisibilité engendre un certain sentiment de confort et de sécurité qui donne naissance à un désir d'exploration et à un regain d'énergie propices à l'épanouissement spirituel. Nous regardons et sentons les biorythmes quotidiens - les rythmes qui font la vie. Dans le monde trépidant qui nous entoure, consacrer du temps à la réflexion intérieure est assurément un luxe, mais c'est également une nécessité.

Une fois qu'on a fait l'expérience directe du cancer, on voit les choses un peu différemment. Cela se traduit peut-être par une sensibilité accrue à la vulnérabilité de la vie, une appréciation approfondie de ce que chaque jour nous apporte ou une passion plus manifeste pour notre emploi. Nous ne tenons plus rien pour acquis. Nous voyons avec nos yeux et nous écoutons avec notre cœur. Mais cela ne se produit que si nous sommes en relation avec notre source spirituelle. Ce faisant, nous permettons à d'autres personnes d'honorer leur propre être spirituel et de rester ouvertes au processus et au mystère de la vie. Renouveler notre spiritualité fait de nous des guérisseurs plus efficaces.

L'unicité de la réaction face à l'expérience du cancer donne naissance à un sentiment d'admiration et d'émerveillement. Les incroyables forces dynamiques sous-tendant la manière dont les patients et les proches transcendent l'expérience du cancer m'amènent immanquablement à penser à la résilience de l'esprit humain. C'est la foi spirituelle animant les familles qui les aide à s'adapter à la vie touchée par le cancer et les pousse à poursuivre leur cheminement. Faites l'effort d'observer, sans rien faire d'autre, et d'apprécier la manière dont chaque esprit danse au rythme de l'âme.

\section{Le ressourcement intérieur}

Avez-vous remarqué à quel point la quête spirituelle est devenue populaire parmi le grand public à l'aube du nouveau millénaire? Durant cette année de transformation, les récits sur le pouvoir de guérison de la prière et la quête de sens se trouvaient souvent à la une

Janice Post-White, RN, PhD, FAAN, est Professeure, Université du Minnesota, Minneapolis. 
des revues populaires et sont encore omniprésentes de nos jours. Quel est donc le but de cette quête?

En tant qu'infirmières en oncologie, nous savons de longue date que si deux personnes sont atteintes de la même maladie (tout en sachant bien qu'une même maladie n'est jamais exactement pareille chez les êtres humains du fait de leur unicité), c'est la personne qui possède en elle le plus de force, de volonté et de désir de surmonter la maladie qui a les plus grandes chances d'avoir des résultats positifs par rapport à la personne qui subit avec apathie les aléas du traitement ou qui a renoncé. C'est la quête de sens qui alimente l'esprit. Et c'est l'esprit qui donne vie.

Lorsqu'ils font face à une maladie qui menace leur vie, les individus sont forcés de réfléchir à qui ils sont et à ce qui compte vraiment pour eux. Certains peuvent se fixer de nouvelles priorités et réaliser les choses qu'ils voulaient vraiment faire et qu'ils avaient mis de côté toute leur vie durant. Le cancer les oblige à écouter leur esprit. Au lieu de réagir aux “j' aurais pu” et aux “j'aurais dû" en provenance du cerveau, ils apprennent à réagir aux "Je veux faire..." qui émanent du cœur. Le cancer éveille le besoin que nous avons d'honorer notre esprit en étant à l'écoute de notre cœur.

Il arrive parfois que notre essence spirituelle ne soit pas si évidente. Le refus défensif manifesté par les adolescents atteints de cancer en réponse à leur colère, à leur rébellion et à leur ressentiment est en réalité leur esprit qui essaie de comprendre la colère, la peur et la peine et d'y donner un sens. Tout cela reflète le combat intérieur en quête de sens. Lorsque les patients n'essaient plus de trouver un sens à ce qui leur arrive, ils renoncent. Alors, comment pouvons-nous aider nos patients dans leur quête de sens spirituel?

Il y a des gens qui trouvent leur essence spirituelle dans la nature, dans la solitude et dans de longues randonnées en forêt ou le long de la mer ou même en restant dans leur propre cour, là où les sens sont uniquement absorbés par le monde environnant. Il y a aussi des gens qui la trouvent à travers d'autres personnes - par le biais de l'énergie de ceux qui participent à l'aventure qu'est la vie. Chacun trouve sa propre façon de rester en contact avec son esprit. Il nous arrive quelquefois d'avoir besoin de rappels pour le faire. Il se peut que nous perdions de vue l'importance d'être qui nous sommes ou alors, nous sommes tellement occupés à faire que nous oublions d'être.

Le partage de notre essence spirituelle peut renouveler notre conscience spirituelle. Un jour, cet été, après avoir enfin appris à chevaucher les vagues, j'ai découvert le pouvoir de guérison de l'océan. Je voulais partager mon expérience avec les parents d'une fillette de cinq ans qui font face à la progression inexorable de l'astrocytome de leur enfant. Je voulais qu'il leur soit donné à eux aussi de ressentir ce pouvoir de guérison. J'ignorais si ce qui revêt énormément de sens pour moi aurait la même résonance pour eux. J'ai pris la chance de partager ma foi spirituelle. Voici ce que je leur ai écrit alors que je me trouvais à plus de 4000 miles d'eux:

Tout ce que je souhaite, c'est que vous soyez ici avec moi en ce moment. Pour sentir le pouvoir de guérison de l'eau turquoise de l'océan lorsqu'on y baigne jusqu'à la poitrine. Imaginez que les vagues élèvent votre être spirituel au-dessus de leurs oscillations, vous bercent au rythme de la marée et noient vos peurs dans les vagues de la planète. Une fois que vous avez appris à lâcher prise, vous accueillez le rythme de la nature et vous laissez l'océan vous masser le corps et l'esprit.

Tandis que vous flottez tous à l'unisson, j'imagine d'autres enfants qui rient et s'amusent comme des fous à pourchasser les vagues (et à se faire pourchasser par elles!) le long du littoral et à faire des platventre dans l'écume des vagues frappant le sable. Vous vous souviendrez alors des joies passées et vous prendrez plaisir à regarder la joie qui enveloppe de nouveau votre famille. Et peut-être même que si les dauphins ont envie de s'amuser, qu'ils feront des tours et des batifolages avec vous pour célébrer la joie et l'épanouissement spirituel qui caractérisent la vie. Voilà ce que je souhaite pour vous, un jour, lorsque le moment sera venu. Je vous envoie mon amour et je souhaite que l'aloha hawaiien soit avec vous (que l'amour pénètre au plus profond de votre être).

Il est pénible de regarder souffrir. Nous voulons supprimer la peine. Le cancer est quelquefois synonyme de solitude, d'incertitude et de frayeur. À travers la souffrance, nous découvrons ce qui compte vraiment et nous trouvons le courage de vivre en harmonie avec cette découverte. Nous utilisons nos forces pour effectuer les changements que nous devons faire afin d'être fidèles à nos valeurs les plus profondes, à notre être spirituel. Mais tout cela n'est pas aussi facile qu'il n'y paraît.

Dans notre étude sur l'espoir (Post-White et al., 1996), nous avons défini la spiritualité comme étant "la quête du sens et du but existentiel de la vie par rapport à soi-même, à autrui et à un être supérieur" (p. 1572). Á l'époque, l'insistance de l'aumônier à vouloir inclure la dimension relationnelle me laissait quelque peu sceptique. Pour moi, la spiritualité représentait un cheminement autonome. Mais depuis, j'ai réalisé que le sentiment de relation à autrui est un élément important de la spiritualité. Le fait de partager notre esprit donne un sens et un but. Quand nous fortifions la vie autour de nous, nous fortifions notre vie intérieure (Remen, 2000).

Lorsque je réfléchis à la signification des relations spirituelles pour les soins infirmiers en oncologie, je me pose des questions sur ce qui est advenu de l'être spirituel en premier lieu. Pourquoi devons-nous essayer de récupérer quelque chose qui n'aurait jamais dû nous quitter? Est-il présent et n'y nous prêtons aucune attention, est-il noyé dans les compressions budgétaires, les pénuries de personnel, la lourdeur des cas et l'intensité des protocoles thérapeutiques? Les liens qui nous reliaient à notre être spirituel se sont-ils dissous et avonsnous besoin de rappels pour le trouver et l'honorer? Qu'ils soient absents ou ne soient pas aussi manifestes qu'ils pourraient l'être aujourd'hui, les liens spirituels vont au-delà des soins professionnels que nous prodiguons à nos patients. Il s'agit de liens plus profonds qui émanent du cour. Ils ne se manifestent pas dans les résultats d'analyses de laboratoire, dans les radiographies ou dans les protocoles de traitement. Non, l'essence spirituelle ne se manifeste que lorsque nous communiquons réellement avec autrui. La présence de l'esprit se révèle à travers les histoires que nous entendons.

Comment parvenons-nous à rester centré et connecté à notre propre esprit et dans une moindre mesure, avec l'esprit de nos patients? Eh bien, nous faisons l'effort d'écouter. Et de réfléchir. Notre esprit sait ce qui constitue l'essentiel dans notre vie. Il faut simplement que nous soyons à l'écoute. Si vous écoutez avec votre cœur, vous entendrez. Et si vous restez ouvertes à l'idée de découverte, vous verrez. La quête de sens nous aide à percer l'obscurité tandis que le jour perce. Elle étaye notre désir de vivre chaque jour de notre cheminement sur terre.

\section{La démarche et le mystère}

Quoique certains aspects de la vie soient prévisibles, cette dernière est un mystère permanent. En honorant la vie en tant que démarche, nous reconnaissons que nous avons besoin de croître. Et en reconnaissant le mystère qui caractérise chacune des phases de notre vie, nous écoutons notre être spirituel.

Nous établissons tous des buts dans la vie. L'atteinte de ces buts est une démarche. Parfois nous les atteignons, et parfois non. Quelquefois, nous nous rendons compte qu'ils ne sont pas réalistes et nous les abandonnons. Lorsque nous atteignons un but particulier, nous célébrons notre réussite et nous en établissons de nouveaux. Nous progressons en tirant profit de nos expériences. Cette démarche nous permet de mieux comprendre qui nous sommes, de découvrir notre être spirituel et d'apprendre ce qui revêt une importance primordiale pour nous.

Il en est de même pour nos patients. Leurs buts ne disparaissent pas parce qu'ils ont le cancer, bien que ce soit ce qui semble se produire, surtout au tout début. Il se peut qu'ils les redéfinissent. $\mathrm{Ou}$ qu'ils les mettent de côté. Ou même encore qu'ils lâchent leur 
emprise. Dans le cadre de la démarche qui amène les gens à comprendre la signification du cancer dans leur vie, certains apprennent que ce qu'ils essayaient de faire n'est pas en harmonie avec leur essence spirituelle. Et ils apprennent à lâcher prise, parfois avec une énorme réticence, parfois avec un énorme soulagement.

Tout comme la démarche accompagnant l'atteinte d'un but nous permet d'approfondir la connaissance que nous avons de nousmêmes, la démarche accompagnant l'espoir nous permet d'aller de l'avant. L'espoir se trouve dans la démarche de questionnement, dans la démarche qui nous amène à compter sur notre autodétermination et sur nos ressources intérieures, dans l'acceptation de l'aide de nos proches, de nos amis et des professionnels de la santé, dans la démarche qui consiste à vivre la vie une journée à la fois et à anticiper l'avenir (Post-White et al., 1996). Ce qui donne vie, ce n'est pas ce que vous attendez de la vie (peu importe les buts que vous vous êtes fixés), mais bien la démarche liée à l'espoir. Ce concept n'est pas toujours facile à saisir, notamment par ceux et celles d'entre nous chez qui l'atteinte du résultat souhaité prime sur tout le reste!

Pourtant, même un enfant de huit ans peut le saisir. Lorsque j'ai demandé à mon fils ce que l'espoir voulait dire pour lui, il m'a répondu ceci: "Que je sais que je peux le faire. Que je peux croire en moi et savoir que je peux surmonter ce problème." Cette perspective lui donnait la force nécessaire pour surmonter tout ce qui entravait son chemin, et pas seulement pour atteindre son but du moment. La démarche qui l'amenait ainsi à croire en lui-même lui donnait l'espoir et la force dont il avait besoin pour faire face à chaque journée, peu importe ce qu'elle lui réservait.

En valorisant la démarche tout autant que le résultat, nous apprenons à être présents dans le moment. Cela nous permet d'entrer en relation avec notre être spirituel et de favoriser le déroulement de la démarche. En étant présent dans le moment et en concentrant son attention sur sa propre réaction, mon fils était amené à prendre luimême le contrôle en laissant la vie évoluer autour de lui. Il avait le pouvoir de déterminer sa propre réaction. Ce n'est pas l'événement en soi qui détermine ce qui se passera par la suite, mais bien la manière dont nous y réagissons.

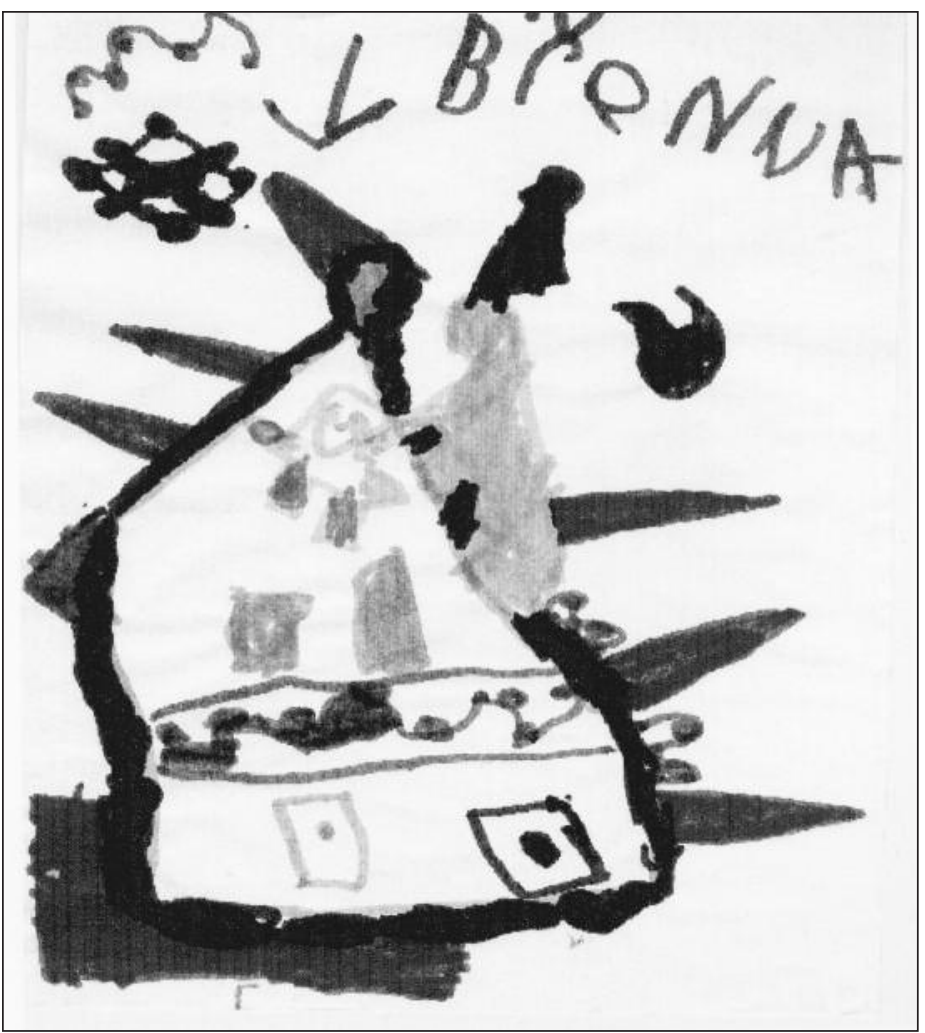

Figure 1
Elizabeth Kubler-Ross a été la première à présenter le cancer en tant que démarche. Elle a avancé que les patients atteints de cancer traversent diverses phases - les phases de déni, de colère, de négociation, de désespoir et d'acceptation (Kubler-Ross, 1997). Nous reconnaissons désormais que la démarche n'est pas la même pour tous. C'est un outil qui nous aide à comprendre la réaction face au cancer en tant que démarche. Il arrive parfois que la démarche ne soit pas manifeste avant que les patients et les proches aient réfléchi à la croissance ainsi vécue et reconnaissent le chemin parcouru. La réflexion apporte une meilleure connaissance de soi et donne un sens.

\section{La réflexion}

C'est en examinant les dessins que Brennan avait réalisés durant sa première année de traitement que j'ai découvert sa perspective, la démarche qu'il avait suivie pour donner un sens à ce qui se passait et pour endurer chaque nouvelle journée en tant que petit enfant de quatre ans atteint de cancer. Cela m'a aidée à apprécier l'expression de ses sentiments et de son subconscient.

Le premier jour qu'il a passé à l'hôpital, il a dessiné l'image d'un petit oiseau jaune enfermé dans une cage (Figure 1). Il y avait aussi des pointes rouges et noires symbolisant la peur et la colère. Il y avait quelque chose à l'extérieur qui voulait entrer à l'intérieur. Un panneau avertissait les gens de se tenir à l'écart. Deux jours plus tard, il a dessiné une seconde cage. Une flèche indiquait aux gens par où entrer. Il a graduellement accordé sa confiance à ses soignants et leur a permis de l'aider. Les couleurs qu'il utilisait étaient vives et il y avait des jouets et des lumières - "pour que ce soit joli pour Maman", avait-il dit. Pour protéger Maman de la peine et de la douleur qu'il ressentait, ai-je pensé. Il a dessiné l'image d'un voilier voyageant au milieu de nuages, d'averses et de rayons de soleil. Un enfant de quatre ans avait compris au plus profond de son être, on ne sait trop comment, que sa vie avait changé du jour au lendemain.

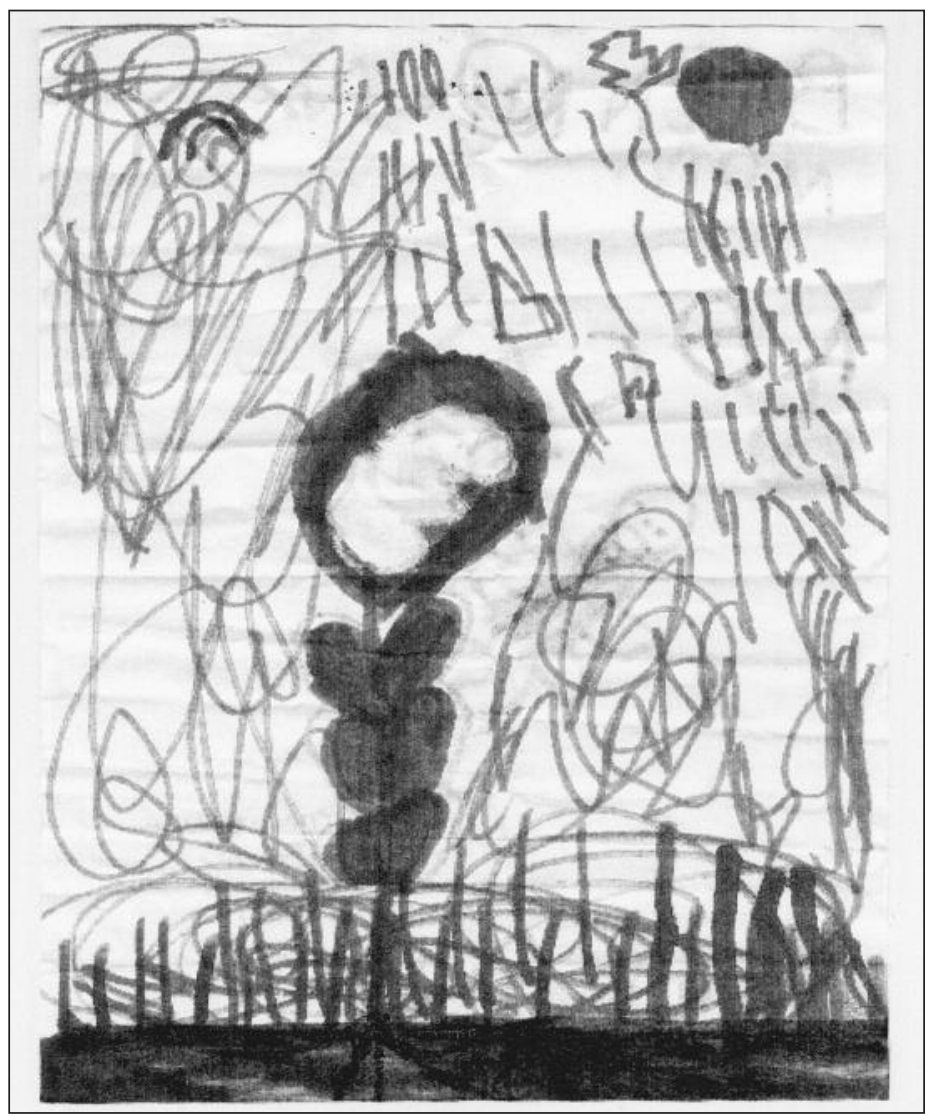

Figure 2 
L'expérience acquise au cours de cette première année de traitement marquée par la phase d'induction, la phase de consolidation, la phase d'intensification différée 1 et 2 et enfin la phase d'entretien a permis à Brennan de réaliser qu'il avait en lui une grande force. Il s'intéressait particulièrement à l'histoire du cavalier sans tête. Après l'avoir lu à de nombreuses reprises, il a dessiné une image du cavalier où il a mis une chandelle à la place de la tête, afin que le cavalier puisse trouver son chemin. J'imagine qu'il avait découvert son pouvoir à force de s'être frayé son propre chemin durant des jours si sombres. Il faisait des dessins de ses os auxquels il ajoutait un cœur rouge et un cerveau noir parce qu'il savait que ces deux organes sont essentiels à la vie. Et durant l'été, alors que nous profitions d'un répit dans son traitement intense, il a dessiné une fleur solitaire de haute taille qui prospérait, entourée de spirales bleues, de gouttes de pluie et de rayons de soleil (Figure 2). Au beau milieu du chaos, le soleil, l'eau et la terre étaient présents pour la nourrir. Il avait plié l'image à la manière d'un éventail et me l'avait remise en s'écriant: "C'est pour toi!" En dépliant avec soin l'image, je redonnais vie à la force qu'elle contenait.

La fin de sa première année de traitement reflétait la progression de son cheminement. Il a dessiné un phare qui guidait le voilier sur lequel il se trouvait en compagnie de son frère. C'était la première fois qu'il incluait son petit frère dans ses dessins. Il sentait que son énergie revenait et qu'un nouveau feu l'habitait. Il dessinait des images d'une maison remplie de flammes, un soleil rouge vif qui embrasait une journée naissante et un arc-en-ciel aux douces couleurs pastel sous lequel volaient trois oiseaux gris. J'ai commencé à percevoir sa démarche, sa croissance au fil des mois. De l'oiseau captif et effrayé, il était devenu un oiseau débordant de vie et avait créé son propre arcen-ciel pour égayer sa vie après les orages qui l'avaient secouée. Si un enfant de cinq ans atteint de cancer peut trouver un tel arc-en-ciel, nous pouvons tous trouver les nôtres. Il faut tout simplement que nous nous rappelions à l'ordre. En étant totalement présentes, nous sommes à même de découvrir notre force intérieure. Cette présence consiste à observer la vie telle qu'elle se déroule et à répondre aux appels de notre être spirituel.

En réfléchissant au chemin parcouru, nous voyons plus clairement où nous nous rendons. Lorsque nous nous hâtons pour aller quelque part, nous ne profitons ni de la beauté du paysage ni des possibilités qui s'offrent à nous le long du chemin. Rachel Naomi Remen (Remen, 2000) aborde le mystère dans son ouvrage My Grandfather's Blessings. Elle raconte comment elle a décidé, en s'attirant les foudres de deux architectes, de mettre la porte principale de sa maison dans un endroit où elle est invisible depuis la rue et où le visiteur ne la découvre qu'après avoir emprunté une allée sinueuse et obscure. Elle décrit comment on entre chez elle, en passant par une grille, en empruntant un escalier, un palier, un autre escalier, en traversant une terrasse et en montant quelques marches de plus pour enfin apercevoir sa porte de devant. La porte n'est pas visible de la rue et pas même des escaliers à pente raide. En revanche, le visiteur verra, en chemin, un arbre magnifique, une roseraie bien entretenue, un panorama époustouflant de la baie de San Francisco et une vue sensationnelle des montagnes qui se profilent à l'arrière de la jolie petite prairie qui lui sert de cour arrière. Sa réflexion sur sa décision l'amène à conclure: "si on sait où on va, on a tendance à ne plus rien voir ni entendre et à nous endormir" (Remen, 2000, p. 289).

Lorsque nous prenons le temps de nous arrêter et de réfléchir, nous voyons souvent les choses sous un angle différent. Combien d'entre nous se sont laissées endormir par la routine? Ratons-nous des messages importants dans la vie quotidienne? Par contre, la démarche de découverte nous amène à vivre le moment présent et à apprécier le mystère. Si nous pouvons accepter dans notre vie une part de mystère, nous pouvons rester à l'écoute de notre être spirituel. Car, quand il n'y a plus de mystère, l'espoir disparaît. N'est-ce pas là une leçon que nous avons apprise de nos patients?

\section{La découverte}

En recherche, les découvertes heureuses faites par hasard illustrent à quel point il importe de rester ouvert au mystère et à la découverte. Habituellement, nous utilisons comme point de départ des hypothèses prescrivant ce que l'on est en droit d'attendre. Nous anticipons où nous allons et ce que nous espérons y trouver. Nous concevons l'étude afin de vérifier ces effets et nous les confirmons ou les infirmons en indiquant une valeur $\mathrm{p}$ supérieure ou inférieure à 0,05 . On nous a appris à voir les choses comme étant bien distinctes, à considérer la cause et l'effet et à répondre aux questions axées sur des hypothèses.

Il arrive parfois que nous perdions de vue la sagesse spirituelle dans la démarche qui consiste à essayer de tout expliquer. Nous choisissons une certaine direction, nous attendant à voir ce que nous anticipons. En recherchant une réponse précise, il se peut que nous passions à côté de résultats qui revêtent peut-être une plus grande importance. Quelquefois, la vraie découverte correspond à ce qui n'a pas été mesuré ou à ce que nous avons mis en évidence au cours de l'étude, et non pas à ce que nous cherchions à déterminer en premier lieu. Le mystère exige parfois que nous renoncions à notre quête de réponses.

La psychoneuroimmunologie est un domaine intrigant caractérisé par le mystère. Au cours des 20 dernières années, les scientifiques ont tenté de découvrir les liens qui existent entre le mental, le corps et l'esprit. Descartes jugeait qu'ils étaient séparés. Mais les récits de guérisons miraculeuses, les histoires de survivants qui n'avaient pourtant aucune chance de s'en sortir, le sentiment de bien-être qui anime certaines personnes en dépit d'une maladie florissante, sont autant de défis à la logique des processus biologiques. Nous savons que le mental et le corps communiquent par l'intermédiaire des neurohormones et des neuropeptides. Nous savons maintenant que certains processus mentaux et certaines émotions, comme la colère et la peur, indiquent des réponses immunitaires, telles qu'une élévation des niveaux d'interféron gamma et une baisse des IL-2 (Post-White et al., 2003). Mais les interactions sont complexes avec des boucles de régulation et des signaux qui "ouvrent" ou "ferment" certains transmetteurs. Plus nous cernons de nouveaux détails, plus nous réalisons que nous n'avons pas la réponse. Notre cheminement présente davantage de questions que de réponses. Les sciences contribuent aux connaissances cliniques et à la pratique spécialisée. À chaque pas que nous faisons, nous pensons que nous avons un meilleur entendement. Mais la vraie connaissance ne se limite pas aux mises à l'essai et aux analyses.

\section{La présence totale}

Souvent, les patients et les familles n'ont pas besoin qu'on leur fournisse des réponses. Ils savent que l'incertitude fait partie intégrante du cancer. Mais ils ont besoin qu'on les rassure et qu'on les écoute afin d'alléger la peur de l'inconnu. Pam McGrath a réalisé des entrevues auprès de 12 familles australiennes dont un enfant était atteint du cancer (McGrath, 2002). Ces familles ont rapporté le stress qui accompagnait l'incertitude, le choc du diagnostic et le sentiment d'être coincés à bord de montagnes russes particulièrement désagréables. Les membres de ces familles ont décrit ce dont ils avaient besoin: la franchise de l'équipe de soins, la possibilité de partager leurs sentiments dans un environnement chaleureux sans que l'enfant ne soit présent, et la reconnaissance du fardeau de la situation. Ils avaient besoin que quelqu'un les accompagne dans leur chagrin, que quelqu'un soit là spirituellement pour eux lorsqu'ils ont besoin d'être rassurés ou d'être écoutés. Quelqu'un qui comprenne la pénibilité de leur vie à ce moment précis.

Nous avons obtenu des résultats similaires lorsque nous avons demandé à des patients adultes atteints de cancer comment les professionnels de la santé leur donnaient de l'espoir (Koopmeiners et al., 1997). Ils nous ont répondu que les soignants le faisaient en étant réellement présents à leurs côtés, en les écoutant, en leur fournissant de l'information et en leur manifestant de la compassion. Souvent, ce 
qui compte, ce n'est pas ce que nous disons ou ce que nous faisons, mais notre présence. En étant réellement présentes, nous communiquons notre désir de les écouter. L'écoute est un puissant outil de guérison. Parfois, ce sont les petites choses que nous faisons qui font toute la différence!

Dans une récente étude (Post-White, Kinney, Bernsten, Savik et Lerner, 2003), nous avons vérifié si à elle seule la présence d'une soignante bienveillante influençait les réactions affectives et les symptômes physiques chez les patients en les comparant à des patients qui recevaient des massages ou des séances de toucher thérapeutique. Chez 230 patients adultes atteints de cancer, nous avons découvert que la présence réelle ne différait pas des soins standards (la période contrôle). Les massages et le toucher thérapeutique, eux, avaient des répercussions significatives et immédiates sur la pression artérielle, le rythme cardiaque, la fréquence respiratoire et la douleur ainsi qu'une incidence à plus long terme (4 semaines) sur l'anxiété, la fatigue, les perturbations de l'humeur et l'utilisation d'anti-inflammatoires non stéroïdiens (PostWhite, Kinney et al., 2003). Nous en avons conclu que notre présence, utilisée en combinaison avec nos interventions, est plus efficace que l'une et l'autre appliquées séparément.

\section{Aider autrui à découvrir son pouvoir intérieur}

Qu'avons-nous d'autre, à part notre présence totale, qui aide les familles dans leur vécu du cancer? Le meilleur cadeau que nous puissions leur faire est de les aider à découvrir le pouvoir qui est en elles et à l'utiliser pour obtenir de l'information, prendre des décisions, mobiliser les ressources et guider leur cheminement. Nous pouvons également les aider à trouver leur force intérieure et les liens qui les unissent à autrui tout au long de leur cheminement.

Quand nous amenons les patients à découvrir leur pouvoir intérieur, nous favorisons la résilience. La résilience est un sentiment de confiance et de maîtrise, un sentiment de confiance en soi et une aptitude à dépasser la situation (Haase et Heiney, 1999). La résilience est un processus dynamique. Elle prend appui sur des compétences d'adaptabilité, de cohésion et de communication. La résilience s'accompagne d'une adaptation de nature combative et coopérative plutôt que de se manifester par des comportements de défense ou d'évitement. Elle fait fond sur la capacité de la personne à découvrir un sens et de l'espoir dans une situation pénible.

Comment favorisons-nous la résilience chez les patients et les proches? Six mois après le diagnostic de Brennan, j'ai dessiné la métaphore du cancer sous forme de périple en voilier (Post-White, 1998). Le voilier quitte le rivage avec à son bord ma famille qui se prépare à la hâte et sans trop savoir quoi faire pour le périple qui l'attend; ma famille est forcée d'apprendre à toute vitesse comment on équilibre la charge, à former une équipe fiable, à faire les relevés nécessaires pour déterminer le parcours vers une destination incertaine, à dompter le vent dans les voiles et à surmonter les tempêtes, tout en focalisant son attention sur le périple qui débute. Ma famille et les autres apprennent aussi à célébrer les journées ensoleillées et à rechercher les arcs-en-ciel qui ornent souvent le ciel après les tempêtes.

Prendre la barre est intimidant. Quel réconfort pour les patients et les familles que de savoir que nous sommes à leurs côtés à chaque instant. La constance est de mise puisqu'ils sont en train d'établir des liens et une relation de confiance. Tout au long de leur périple, nous les aidons à découvrir leur propre pouvoir en soufflant dans leurs voiles. Un voilier n'avance pas s'il ne profite pas d'un vent doux mais constant. Si vous ne savez pas être le vent, pensez au rôle du remorqueur qui tire à tout coup une charge plus lourde que lui-même et l'emmène dans la direction où il pense qu'elle devrait aller. Imaginez un peu ce que ressent la famille que vous remorquez!

Revenez un peu en arrière et imaginez que vous êtes le vent qui gonfle les voiles et propulse doucement le voilier dans son cheminement. Bien sûr, il faut que les voiles soient en place et que le patient et ses proches soient prêts pour le voyage. Nous les accompagnons dans leur périple et nous apprenons quand il convient de souffler plus fort et quand il est préférable que nous les observions sans rien faire. Nous les aidons à naviguer des eaux inconnues en les préparant à relever les défis qui s'annoncent. Nous n'ignorons pas que les tempêtes perturbent fortement les voyages à la voile et nous apprenons à demeurer en relation avec notre être spirituel afin de mieux guider le leur et lui répondre. Nous fêtons les journées ensoleillées et nous les encourageons lorsque les nuages s'amoncellent. Nous sommes réellement présentes aux côtés des patients survivant au cancer et des proches pleins de résilience qui tracent leur itinéraire sur la carte.

La raison d'être de notre compassion est d'aider les patients et les proches à découvrir le pouvoir qu'ils ont en eux. Nous ne pouvons pas éclaircir le mystère de la vie au nom des familles dont nous croisons le chemin. Il faut qu'elles en fassent l'expérience elles-mêmes. Le soin, la compassion, ce n'est pas faire quelque chose pour autrui, c'est être réellement présent et devenir le vent qui gonfle les voiles. Tout comme les grands enseignants savent faire découvrir à leurs élèves le pouvoir qu'ils ont en eux en les motivant à apprendre, nous aidons les familles, non pas en faisant certaines choses pour elles, mais en les aidant à régler leurs voiles pour profiter au maximum du vent et poursuivre leur périple. Elles seront ainsi mieux préparées face aux exigences de la maladie qu'elles ne manqueront pas de rencontrer sur leur parcours.

Comment pouvons-nous apporter notre aide? Tous les patients et tous les aidants naturels remettent en question leurs aptitudes à un moment ou à un autre. Tous les soignants se demandent un jour s'ils ne devraient pas faire davantage ou ils se sentent dépassés par ce qu'ils font et se sentent coupables de ne pas faire plus. Ils ont besoin qu'on les rassure - qu'on les rassure véritablement - en leur disant qu'ils font du bon travail. Il arrive parfois que nous soyons trop préoccupées par nos propres tâches pour les féliciter du travail qu'ils accomplissent. Les proches ont besoin d'entendre des mots tels que "Vous faites vraiment du bon travail!"

Les familles gagnent en expertise et manifestent une grande ingéniosité pour trouver les réponses et pour s'orienter. Mais le soin est une énorme responsabilité, une responsabilité qui épuise facilement la vitalité spirituelle et les sources d'énergie. Le cancer est un périple familial. La présence de la famille est plus manifeste en oncologie pédiatrique, mais il est tout aussi important de reconnaître, entraîner et soutenir les aidants naturels en oncologie de l'adulte.

Je me souviens encore très bien de la première fois où l'on m'a demandé: "Et comment va la maman?" C'était huit mois après le diagnostic de Brennan. C'était la première qu'on me demandait comme j'allais, moi. Une fois posée, cette question toute simple en apparence m'a permis de prendre un peu de recul et d'évaluer l'état dans lequel je me trouvais réellement. J'ai pris conscience du fait que je pouvais penser à moi un instant et qu'il importait que je le fasse. Cette question validait aussi l'importance de mes sentiments. Ou alors j'ai ressenti du réconfort en sachant que quelqu'un s'inquiétait suffisamment de moi pour me demander comment j'allais. Quelque chose qui semble si simple peut être si puissant.

Il nous est plus facile d'aider les familles à découvrir le pouvoir qui est en elles lorsque nous connaissons leurs forces et leurs besoins. Dans l'étude de McGrath (2002), les soignants familiaux indiquaient que la compréhension que les professionnels de la santé avaient de leurs propres besoins était de la plus grande utilité pour ceux et celles qui s'occupent d'un proche atteint du cancer. Ils ont ajouté que cela les aidait beaucoup lorsque les soignants professionnels reconnaissaient la difficulté de la situation et les soutenaient en appuyant les efforts d'adaptation qu'ils fournissaient, peu importe leur nature. Les proches ont besoin qu'on les rassure même si on croit que ce qu'ils font est loin de l'idéal qu'on pense qu'ils devraient atteindre. 


\section{Le ressourcement de l'esprit}

À la American Hospital Association, la plus haute priorité en matière de pénurie de personnel infirmier est de promouvoir un environnement de travail significatif (American Hospital Association, 2002). N'oubliez pas ce qui donne du sens à votre travail. Maintenez la dimension spirituelle même au beau milieu des crises externes qui frappent les soins de santé autour de vous (voir le tableau 1). La manière dont nous réagirons face à ces crises déterminera notre degré de réussite.

Imaginez un peu que vous êtes Tiger Woods. C'est bien là une personne qui me fascine. Eh oui, j'admire l'incroyable force et l'incroyable précision qui caractérisent ses décoches de 300 mètres qui vont droit au but. Mais plus important encore, j'admire la sagesse intérieure qui émane de lui, le calme avec lequel il fait face à d'incroyables pressions et sa capacité à vivre le moment présent et à se concentrer. Comment y parvient-il? Grâce à un entraînement, une détermination et un esprit incroyables. En réponse à la question cidessus, il a déclaré: "Je crois que les gens ont tendance à rendre les choses simples plus compliquées qu'elles ne le sont vraiment. Pour moi, tout est question d'équilibre. J'essaie simplement de faire en sorte que les choses demeurent aussi simples et aussi équilibrées que possible" (Newport, 2002, p. 139).

Le golf a l'air simple quand Tiger Woods le pratique. Malgré les perceptions d'aucuns, le golf consiste avant tout à savoir renoncer au pouvoir et au contrôle et à trouver l'équilibre et le rythme dans l'élan. Peut-être que la vie est comme le golf en ce qu'il faut savoir renoncer au pouvoir et au contrôle et trouver l'équilibre et le rythme dans sa vie. Lorsque nous sommes en harmonie avec notre être spirituel, nous sommes ouverts au mystère continuel de la vie. Le mystère a le pouvoir de mettre au jour l'émerveillement, la joie et la vitalité. Les gens qui s'émerveillent ne sont jamais victimes de surmenage (Remen, 2000). En faisant place au mystère et à la découverte dans votre vie, vous développerez votre esprit de compassion.

Le mystère se trouve dans la manière dont nos patients résistent jusqu'au dernier moment, en espérant un miracle de la science. Lorsque le mystère disparaît, l'espoir se volatilise. Nos patients ont appris une nouvelle façon de vivre . Nous pouvons le faire aussi.

\section{Références}

American Hospital Association, Commission of Workforce. (2002). In our hands: How hospital leaders can build a thriving workforce. [On-line]. Available: www.aha.org

Haase, J.E., \& Heiney, S.P. (1999). Research triangulation to derive meaning-based quality-of-life theory: Adolescent resilience model and instrument development. International Journal of Cancer, 12(Suppl.), 125-131.

Koopmeiners, L., Post-White, J., Gutknecht, S., Ceronsky, C., Nickelson, K., Drew, D., et al. (1997). How health care professionals contribute to hope in patients with cancer. Oncology Nursing Forum, 24(9), 1507-1513.

Kubler-Ross, E. (1997). On death and dying (reprinted). NY: Simon \& Schuster.

McGrath, P. (2002). Beginning treatment for childhood acute lymphoblastic leukemia: Insights from the parents' perspective. Oncology Nursing Forum, 29(6), 988-996.

Newport, J.P. (2002). A sense of who he is. T \& L Golf, 26, 102-107, 132-139.

Post-White, J. (1998). Wind behind the sails: Empowering our patients and ourselves. Oncology Nursing Forum, 25(6), 10111017.

Post-White, J., Ceronsky, C., Kreitzer, M.J., Nickelson, K., Drew, D., Watrud Mackey, K., et al. (1996). Hope, spirituality, sense of coherence, and quality of life in patients with cancer. Oncology Nursing Forum, 23(10), 1571-1579.
Post-White, J., Hannahan, A., Schroeder, L., Johnston, M.K., Salscheider, N., \& Grant, N. (2003). Psychoimmune response to imagery and support in breast cancer survivors. Manuscript in preparation.

Post-White, J., Kinney, M.E., Bernsten, J., Savik, K., \& Lerner, I. (2003). Effects of massage therapy and Healing Touch in cancer. Manuscript submitted for publication.

Remen, R.N. (2000). My Grandfather's blessings. NY: Riverhead Books/Penguin Putnam.

\section{Tableau 1: \\ Le pouvoir de l'esprit}

Le pouvoir du mental

Le pouvoir de l'esprit,

Tout comme le pouvoir de l'océan,

$\mathrm{N}$ 'accepte comme unique vérité

Que ce qui est dans le plus profond de notre être.

Le mental essaie parfois de supplanter l'esprit

Et de capturer le coeur

Afin de donner un sens à la vie.

Mais le pouvoir du mental

Ne parle pas à travers lui-même et le savoir

Mais en connexion avec l'esprit.

Ce n'est pas quand le mental parle que l'esprit écoute

À la façon d'un enfant qui écoute ses parents.

C'est quand l'esprit parle

Que le mental doit être tranquille

Et écouter.

Dès qu'on devient trop à l'aise avec la vie

Et qu'on accepte aisément l'état des choses

Les vagues de l'océan déferlent sur le rivage

En assénant leur message

Pour nous rappeler le pouvoir qui réside à l'intérieur.

Notre esprit fait tout pour nous rappeler qu'il existe Dans notre quête de succès, de bonheur et d'amour Ce n'est pas à nous que nous devons le bonheur.

Lorsque les liens avec notre esprit se relâchent

Plus nous essayons de réussir notre vie

Plus nous risquons la désillusion.

C'est un peu comme l'élan au golf

Plus on s'y essaye et plus notre précision empire.

En essayant de forcer la vie à correspondre

à ce que nous voulons qu'elle soit

Nous nous éloignons de notre esprit.

La vie, c'est la recherche d'équilibre

Et les liens qu'on entretient avec sa source spirituelle.

Découvrez le mystère de votre être spirituel

Et partagez-le

Votre don est dans l'Amour (l'Aloha hawaiien) que vous apportez aux autres

Voilà la source de votre pouvoir

Le mystère intérieur de votre esprit,

Qui fait de vous un être unique. 